\title{
Isolated and combined effects of photobiomodulation therapy, topical nonsteroidal anti-inflammatory drugs, and physical activity in the treatment of osteoarthritis induced by papain
}

Shaiane Silva Tomazoni

Ernesto Cesar Pinto Leal-Junior

Lúcio Frigo

Rodney Capp Pallotta

Simone Teixeira

Patricia de Almeida

Jan Magnus Bjordal

Rodrigo Álvaro Brandão Lopes-Martins 


\title{
Isolated and combined effects of photobiomodulation therapy, topical nonsteroidal anti-inflammatory drugs, and physical activity in the treatment of osteoarthritis induced by papain
}

\author{
Shaiane Silva Tomazoni, ${ }^{a}$ Ernesto Cesar Pinto Leal-Junior, ${ }^{b}$ Lúcio Frigo, ${ }^{c}$ Rodney Capp Pallotta, ${ }^{a}$ \\ Simone Teixeira, ${ }^{a}$ Patricia de Almeida, ${ }^{a}$ Jan Magnus Bjordal, ${ }^{d}$ and Rodrigo Álvaro Brandão Lopes-Martins ${ }^{e, f, *}$ \\ aUniversity of São Paulo (USP), Laboratory of Pharmacology and Experimental Therapeutics, Department of Pharmacology, Institute of Biomedical \\ Sciences, Avenida Lineu Prestes 1524, Butantan, São Paulo 05508-900, Brazil \\ ${ }^{b}$ Nove de Julho University (UNINOVE), Post Graduate Program in Biophotonics Applied to Health Sciences and Post Graduate Program in \\ Rehabilitation Sciences, Rua Vergueiro 235, São Paulo 01504-001, Brazil \\ 'Cruzeiro do Sul University (UNICSUL), Biological Sciences and Health Center, Avenida Doutor Ussiel Cirilo 225, São Paulo 08060-070, Brazil \\ dUniversity of Bergen, Department of Global Public Health, Physiotherapy Research Group, PO Box 7804, Bergen, Norway \\ eUniversidade de Mogi das Cruzes (UMC), Biomedical Engineering Research and Post-Graduate Center, Avenida Doutor Cândido Xavier de \\ Almeida e Souza, 200 Mogi das Cruzes, São Paulo 08780-911, Brazil \\ fUniversidade Camilo Castelo Branco, Faculty of Medicine, Avenida dos Expedicionários Brasileiros, 781, Fernandópolis, São Paulo 15600-000, \\ Brazil
}

\begin{abstract}
Osteoarthritis (OA) is a chronic inflammatory disease and is characterized as a degenerative process. This study aimed to evaluate and compare the effects of a topical nonsteroidal anti-inflammatory drug (NSAID), physical activity, and photobiomodulation therapy (PBMT) applied alone and/or in combination between them in an experimental model of knee OA. OA was induced by injection of papain in the knees of rats. After 21 days, the animals started to be treated with the above treatment. Histological analysis shows that the experimental model of OA induction causes morphological changes consistent with the disease, and among treatments, the PBMT is the most effective for reducing these changes. Moreover, the results demonstrate that PBMT and NSAID reduce the total number of cells in the inflammatory infiltrate $(p<0.05)$ and PBMT was the most effective for reducing the activity of myeloperoxidase $(p<0.05)$. Finally, we observed that both NSAID and PBMT were effective for reducing the gene expression of MMP-3 $(p<0.05)$, but in relation to the gene expression of MMP-13, PBMT was the most effective treatment $(p<0.05)$. The results of this study indicate that PBMT is the most effective therapy in stopping disease progression, and improving inflammatory conditions observed in OA. ๑ 2016 Society of Photo-Optical Instrumentation Engineers (SPIE) [DOI: 10.1117/1.JBO.21.10.108001]
\end{abstract}

Keywords: osteoarthritis; papain; anti-inflammatory drugs; swimming; photobiomodulation therapy.

Paper 160240R received Apr. 13, 2016; accepted for publication Sep. 19, 2016; published online Oct. 18, 2016.

\section{Introduction}

Osteoarthritis (OA) is the most commonly found form of arthritis in humans, ${ }^{1}$ characterized as a degenerative disease that affects the joints as a whole, ${ }^{2}$ including the articular cartilage, subchondral bone, ligaments, joint capsule, synovial membrane, and periarticular muscles. ${ }^{3}$ The pathological effects on these structures are manifested by morphological, biochemical, and biomechanical alterations. ${ }^{4} \mathrm{OA}$ is characterized by a plethora of inflammatory mediators and cytokine production beside leukocyte infiltration, especially neutrophils. ${ }^{4,5}$ Additionally, signs of cartilage degeneration, such as the activation of matrix metalloproteinases (MMPs), are involved in important aspects of tissue damage, leading to the progressive destruction of the joint adjacent structures. ${ }^{4}$

The standard algorithm for OA treatment is to control symptoms, especially pain, prevent progression of the disease, minimize disability, and improve the quality of life of patients. ${ }^{6}$ This algorithm can be divided into three main steps: pharmacological treatment, surgery, and nonpharmacological treatments that may

*Address all correspondence to: Rodrigo Álvaro Brandão Lopes-Martins, E-mail: ralopesmartins@gmail.com be applied alone or in combination. ${ }^{2}$ Currently, pharmacological treatment is the most commonly used and indicated by clinicians to achieve pain relief in mild to moderate cases of OA. ${ }^{7}$ However, Bjordal et al. ${ }^{8}$ clearly demonstrated in a meta-analysis including 13,000 patients that nonsteroidal anti-inflammatory drugs (NSAIDs) and other pharmacological treatments are not different from a placebo concerning OA pain control. In addition, the long-term use of pharmacological treatments causes a wide range of important side-effects. ${ }^{8,9}$ As an adjunctive treatment for OA, the prescription of moderate exercise is very popular, and the most frequently recommended activities are swimming, dancing, hiking, and cycling. The benefits of this nonpharmacological treatment include the reduction of comorbidities frequently associated with OA, delayed physical and functional limitations, and the preservation of healthy joints. ${ }^{10}$

On the other hand, the use of photobiomodulation therapy (PBMT) in OA treatment has achieving increasing evidence and power in recent years. PBMT has proven to be an interesting and efficient alternative to pharmacological treatments to OA. It has been demonstrated that PBMT reduces the knee acute

$1083-3668 / 2016 / \$ 25.00$ (c) 2016 SPIE 
inflammation, ${ }^{11,12}$ reduces cartilage tissue degeneration, and possibly improves tissue repair in OA. ${ }^{12-16}$

It is believed that the combination of more than one therapy in the treatment of musculoskeletal disorders such as OA would potentiate the beneficial effects observed with each single treatment (e.g., NSAID, exercise, and PBMT). However, to date there are no studies that evaluate the effects of these three important therapies prescribed to treat OA isolated, but especially in combination. It still not known if the use of combined therapy actually results in a better prognostic when compared to a single treatment.

In this context, the aim of this study was to evaluate the effects of PBMT, exercise, and topical NSAID alone and as combined therapies, on morphological, inflammatory infiltrate, and related biochemical markers of inflammation and joint degeneration in an experimental model of OA.

\section{Material and Methods}

\subsection{Animals}

Fifty-four male Wistar rats weighing about 200 to $250 \mathrm{~g}$ from the central animal facility of the Institute of Biomedical Sciences, University of São Paulo (USP) were used. The animals were kept in standard conditions of temperature $\left(22\right.$ to $\left.24^{\circ} \mathrm{C}\right)$, relative humidity (40\% to $60 \%$ ), and light and dark cycles of $12 \mathrm{~h}$, with food and water ad libitum. Experimental protocol was approved by the Ethics Committee for Animal Experimentation of the Institute (number 077, page 130, book 02) of Biomedical Sciences, University of São Paulo (ICB-USP).

\subsection{Experimental Groups}

The animals were randomly divided into experimental groups of six animals as follows.

- Control group: healthy animals not submitted to any procedure or treatment.

- OA: induction of OA and nontreated.

- OA + PBMT group (PBMT): induction of OA and treated with PBMT.

- $\mathrm{OA}+$ exercise group (Exerc): induction of OA and treated with swimming training.

- OA + topical sodium diclofenac group (Diclo): induction of OA and treated with topical sodium diclofenac.

- $\mathrm{OA}+$ exercise + PBMT group (Exerc + PBMT): induction of OA and treated with the combination of swimming and PBMT.

- OA + topical sodium diclofenac + PBMT group (Diclo + PBMT): induction of OA and treated with topical sodium diclofenac sodium and PBMT.

- $\mathrm{OA}+$ exercise + topical sodium diclofenac group (Exerc + Diclo): induction of OA and treated with the combination of swimming and diclofenac.

- $\mathrm{OA}+$ exercise + topical sodium diclonefac + PBMT group (Exerc + Diclo + PBMT): induction of OA and treated with swimming, diclofenac, and PBMT.

All treatments used to the experimental groups were initiated 21 days after the last injection of papain and occurred once a day, 3 times a week (every other day) for 8 consecutive weeks for a total of 24 therapy sessions.
After eight consecutive weeks of treatment, animals were euthanized by cervical dislocation $72 \mathrm{~h}$ after the last treatment, in order to collect biological material (intra-articular joint lavage and tissue) for further analysis.

\subsection{Procedures}

Induction of knee OA: the animals were anesthetized with a mixture of ketamine and xylazine $(90$ and $10 \mathrm{mg} / \mathrm{kg}$, respectively; König, Avellaneda, Argentina) intraperitoneally injected. Subsequently, they were submitted to induction of $\mathrm{OA}$ in the right knee, according to Murat et al. ${ }^{17} \mathrm{~A}$ total of $0.2 \mathrm{~mL}$ of papain solution $4 \%, 0.1 \mathrm{~mL}$ of $0.03 \mathrm{M}$ cysteine (activator) were injected intra-articularly with a microsyringe on the right knee of the animal. The needle bevel was positioned medial to the patellar ligament, performing the injection toward the distal articular surface of the femur. This procedure was repeated in the fourth and seventh days after the first application.

\subsection{Treatments}

\subsubsection{Isolated therapies}

- NSAIDs application: Sodium diclofenac gel was used $\left(10 \mathrm{mg} / \mathrm{g}-\mathrm{EMS}^{\circledR}\right.$, Santo André, São Paulo, Brazil), with a dose of $10 \mathrm{mg} / \mathrm{knee}$ applied to the affected knee.

- Physical exercise protocol: The exercise protocol used was adapted from the study of Lima et al. ${ }^{18}$ For the swimming training, we used a glass tank $100 \times 50 \times 75 \mathrm{~cm}^{3}$, filled with water with a temperature of $31^{\circ} \mathrm{C}$. The animals were submitted to a physical exercise protocol of swimming, with the first 2 weeks designed to adapt the animals to physical activity, while the other 6 weeks were assigned to the training itself. The application of the exercise protocol was in accordance with the aforementioned experimental groups. The adaptation and training program has occurred, according to Table 1.

- PBMT application: PBMT parameters used were based on the study Pallotta et al. ${ }^{11}$ We used a laser diode with a wavelength of $830 \mathrm{~nm}$ (infrared) continuously, spot area of $0.028 \mathrm{~cm}^{2}$, power of $100 \mathrm{~mW}$, power density of $35.71 \mathrm{~W} / \mathrm{cm}^{2}$, energy density of $214,2 \mathrm{~J} / \mathrm{cm}^{2}$, energy of $6 \mathrm{~J}$ per point, $60 \mathrm{~s}$ per point, and 1 point on the OA joint. To irradiate the animals, the spot was kept in direct contact with the skin.

\subsubsection{Association of therapies}

All combined therapies were applied in the same way and with the same parameters used in therapies applied in isolation.

- Exercise associated with PBMT: We conducted the exercise protocol, followed by PBMT.

- Diclofenac associated with PBMT: Topical diclofenac was applied, followed by PBMT.

- Exercise associated with NSAID: We conducted the exercise protocol, followed by application of topical diclofenac.

- Exercise associated with NSAIDs and PBMT: We conducted the exercise protocol, followed by application of NSAIDs, followed by PBMT. 
Table 1 Exercise protocol.

\begin{tabular}{|c|c|c|c|c|c|}
\hline Week & Day & Series & Active/recovery & Overload & Total time (min) \\
\hline \multicolumn{6}{|c|}{ Adaptation protocol } \\
\hline \multirow[t]{3}{*}{ First week } & First day & 5 & $1 \min : 1 \min$ & $3 \%$ body weight & 10 \\
\hline & Second day & 10 & $1 \mathrm{~min}: 1 \mathrm{~min}$ & $3 \%$ body weight & 20 \\
\hline & Third day & 8 & $1 \min : 1 \min$ & $5 \%$ body weight & 16 \\
\hline \multirow[t]{3}{*}{ Second week } & Fourth day & 10 & $1 \mathrm{~min}: 1 \mathrm{~min}$ & $7 \%$ body weight & 20 \\
\hline & Fifth day & 10 & $1 \mathrm{~min}: 1 \mathrm{~min}$ & $9 \%$ body weight & 20 \\
\hline & Sixth day & 10 & $1 \mathrm{~min}: 1 \mathrm{~min}$ & $10 \%$ body weight & 20 \\
\hline \multicolumn{6}{|c|}{ Training protocol (swimming) } \\
\hline Third week & - & 11 & $1 \min : 1 \min$ & $10 \%$ body weight & 22 \\
\hline Fourth week & - & 12 & $1 \min : 1 \min$ & $10 \%$ body weight & 24 \\
\hline Fifth week & - & 13 & $1 \min : 1 \min$ & $10 \%$ body weight & 26 \\
\hline Sixth week & - & 14 & $1 \min : 1 \min$ & $10 \%$ body weight & 28 \\
\hline Seventh week & - & 15 & $1 \mathrm{~min}: 1 \mathrm{~min}$ & $10 \%$ body weight & 30 \\
\hline Eighth week & - & 15 & $1 \mathrm{~min}: 1 \mathrm{~min}$ & $10 \%$ body weight & 30 \\
\hline
\end{tabular}

\subsection{Collection of Biological Material}

All animals were anesthetized once intraperitoneally with a mixture of ketamine and xylazine (90 and $10 \mathrm{mg} / \mathrm{kg}$, respectively), and the biological material (intra-articular lavage and joint tissue) was extracted:

- Intra-articular lavage: Animals were anesthetized with a mixture of ketamine and xylazine (90 and $10 \mathrm{mg} / \mathrm{kg}$, respectively; König, Avellaneda, Argentina) administered intraperitoneally. The intra-articular lavage was obtained by injection and aspiration in a final volume of $300 \mu \mathrm{L}$ $(3 \times 100 \mu \mathrm{L})$ of PBS solution + EDTA $(4 \mu \mathrm{M})$.

- Joint tissue collection: The animals were anesthetized with a mixture of ketamine and xylazine (90 and $10 \mathrm{mg} / \mathrm{kg}$, respectively; König, Avellaneda, Argentina) intraperitoneally. Joint tissue was obtained through a cut in the knee region, exposing the joint of the animal.

\subsection{Analysis}

Histology by optical light microscopy: The method used for assembly of the histological slides was based on the study of Murat el al. ${ }^{17}$ Knee joint samples were washed with saline and fixed in $10 \%$ neutral buffered formalin. Subsequently, the samples were decalcified with aqueous $10 \%$ formic acid and embedded in paraffin for forming cuts $4 \mu \mathrm{M}$. Once the preparations of the samples were carried out, the sections were placed on glass slides to be stained with hematoxylin and eosin. After staining, the sections were mounted in permanent blades for further examination with the optical microscope (Nikon ${ }^{\circledR}$, Japan). Subsequently, the sections were photographed by means of a photomicrograph camera. Photographs were taken of all experimental groups using the original magnification $100 \times$. The aspects evaluated were: articular surface, cellularity, and general structure of cartilage.

Total cell count: Aliquots of the intra-articular wash were centrifuged at a speed of $1500 \mathrm{rpm}$ for $10 \mathrm{~min}$ until a cell pellet formation. The pellet was resuspended in 200 of PBS solution + $4 \mu \mathrm{M}$ EDTA $+0.1 \%$ BSA. For total cell count, $20 \mu \mathrm{L}$ was used intra-articular washed diluted with Turk's solution $(380 \mu \mathrm{L})$ and a Neubauer chamber coupled to an optical microscope (Carl Zeiss) with a $40 \times$ magnification. The results were expressed as the total number of leukocytes per joint cavity.

Myeloperoxidase (MPO) activity analysis: MPO is a specific neutrophil enzyme used as an indirect indicator of neutrophil infiltration in the tissue. The synovial membrane was homogenized in $0.5 \%$ HTAB (Sigma Chemical Co.), using $1 \mathrm{~mL}$ buffer per $50 \mathrm{mg}$ of tissue. The homogenate was placed in Eppendorf tubes and heated in an oven for $2 \mathrm{~h}$ at $60^{\circ} \mathrm{C}$ for inactivation of the endogenous catalase activity. Subsequently, the tubes were centrifuged at $12,000 \mathrm{~g}$ for $5 \mathrm{~min}$. Then, $10 \mu \mathrm{L}$ of supernatant was pipetted (in duplicate) in a microplate of 96 wells and added to $200 \mu \mathrm{L}$ of a potassium phosphate buffer solution ( $\mathrm{pH}$ 6.0) containing $0.164 \mathrm{mg} / \mathrm{mL}$ o-dianisidine (dihydrochoride) (Sigma Chemical Co.) and $0.0005 \%$ hydrogen peroxide (Merck, Germany). The level of absorbance was measured in an ELISA reader (Espectra max plus 384) at $460 \mathrm{~nm}$ during 20 min with recordings made at 20 -s intervals. Graphs show the function of variation in absorbance time and $V_{\max } / \mathrm{s}$ (maximum reaction speed per second was calculated from these graphs). The time was measured from the absorbance demonstrated linearity of the variation observed in the graph (optical density or absorbance) versus time in seconds, obtaining a value 
Table 2 Following primer.

\begin{tabular}{ll} 
Primer & \multicolumn{1}{c}{ Following primer } \\
\hline HPRT & Forward: AAGCTTGCTGGTGAAAAGGA \\
& Reverse: TGATTCAAATCCCTGAAGTGC \\
MMP-3 & Forward: CCCGTTTCCATCTCTCTCAA \\
& Reverse: GTGGGTACCACGAGGACATC \\
MMP-13 & Forward: GATGGGCCTTCTGGTCTTCT \\
& Reverse: GAGCTGCTTGTCCAGGTTTC \\
\hline
\end{tabular}

$V_{\max } / \mathrm{s}$. The MPO activity is defined as the degradation micromol of $\mathrm{H}_{2} \mathrm{O}_{2}$ per minute and was expressed as MPO units (UMPO/g).

Analysis of MMP-3 gene expression and MMP-13 by realtime RT-PCR: The sample material was articular cartilage. Total RNA was extracted using Trizol reagent (Gibco BRL) according to manufacturer's instructions. After treatment with DNase, the synthesis of cDNAs was processed by the method of using the SuperScript reverse transcriptase enzyme (Invitrogen) from $2 \mu \mathrm{g}$ of total RNA in the presence of a mixture of random primers and oligo dT. RT-PCR experiments were programmed as follows: an initial one cycle of denaturation for $10 \mathrm{~min}$ at $95^{\circ} \mathrm{C}$ and 40 cycles of amplification $\left(30 \mathrm{~s}\right.$ denaturation at $95^{\circ} \mathrm{C}$ and 1 min annealing and extension at $60^{\circ} \mathrm{C}$ ). The sequences of the primers were the same used by Wang et al. ${ }^{19}$ and are shown in Table 2. The results were interpreted using the $\Delta \Delta \mathrm{Ct} 2$-formula (Ct: number of cycles needed to reach the fluorescence threshold above background value - background) that relates the expression of the gene of interest compared the expression of the HPRT gene control.

\subsection{Statistical Analysis}

Initially, data were tabulated and assessed for normality using the Shapiro-Wilk test, as a normal distribution was obtained and was used for analysis of variance (ANOVA) followed by the Tukey applied for multiple comparisons. Results were expressed as mean and standard error of the mean $( \pm \mathrm{SEM})$ and $p$ values $<0.05$ were considered statistically significant.

\section{Results}

\subsection{Histology by Optical Light Microscopy}

Figure 1 shows the morphological characteristics of the healthy joint (without OA induction), the joint affected by OA (no treatment), and groups treated with exercise, topical NSAIDs, and PBMT in isolation or associated. We note that the experimental model of OA induction causes morphological changes consistent with the disease, and among treatments, the PBMT is the most effective treatment for reducing these changes.

\subsection{Total Cell Count}

Figure 2 shows the total amount of cells in the articular cavity of the animals in all groups. The results demonstrate that the experimental induction model of OA causes an increase in the total number of cells, wherein the PBMT as well as topical NSAID reduce the total number of cells in the inflammatory infiltrate.

\subsection{Myeloperoxidase Activity Analysis}

In Fig. 3, we observed MPO activity in the articular tissue of all groups, it being possible that the experimental model of OA induction caused increased MPO activity, whereas treatment with PBMT was the most effective for reducing the activity of this enzyme.

\subsection{Gene Expression of MMP-3 and MMP-13 by $R T-P C R$}

In Fig. 4, we find the gene expression levels of MMP-3 and MMP-13 in the joint tissue from all experimental groups, which themselves had increased after induction by our experimental OA model. We observed that both the NSAID and PBMT were effective for reducing the gene expression of MMP-3, however, in relation to the gene expression of MMP-13, PBMT was the most effective treatment.

\section{Discussion}

To the best of our knowledge, this study is the first to analyze the effects of physical exercise, topical NSAIDs, and PBMT, used in isolation or combination, as the most effective therapy for reducing morphological alterations, degradation signs, and inflammation resulting from OA.

Our results demonstrated that the experimental model of OA induction using papain triggered significant morphological alterations, which is consistent with the clinical presentation of $\mathrm{OA},{ }^{13,17}$ including irregular joint surfaces, fissure areas, heterogeneously distributed chondrocytes, and bone tissue invagination. Furthermore, our experimental model triggered an inflammatory process in the joint, with the release of inflammatory cells to the articular cavity, and increased myeloperoxidase activity.

Our results indicated that PBMT was the most effective therapy for controlling the clinical signs and morphological alterations caused by OA, and the outcomes were similar to those observed in a healthy joint, as reported by Alves et al. ${ }^{13}$ Similarly, PBMT and topical NSAID were the most effective therapies for decreasing the total cell count in the intra-articular lavage, confirming the results obtained in the histological analysis. PBMT and PBMT associated with physical exercises were effective therapies for reducing MPO activity which is an important inflammatory marker. ${ }^{20}$ Furthermore, our results indicated that PBMT had a major role in the modulation of the inflammation caused by OA by decreasing the important inflammatory markers and possibly contributing to the reduction in disease progression.

Confirming our results with those of Da Rosa et al. ${ }^{15}$ on the effectiveness of PBMT in modulating the inflammatory process, reducing morphological alterations, and decreasing tissue degradation resulting from $\mathrm{OA}$, we observed that PBMT was effective for reducing the inflammatory marker levels and increasing repair. Similarly, Alves et al. ${ }^{13}$ indicated that PBMT was effective in repairing the joint tissue and decreasing joint degradation. Furthermore, Alves et al. ${ }^{14}$ observed that PBMT modulated the inflammatory process, and Oshima et al. ${ }^{21}$ and Dos Santos et al. ${ }^{16}$ demonstrated that PBMT decreased the inflammatory marker levels. 

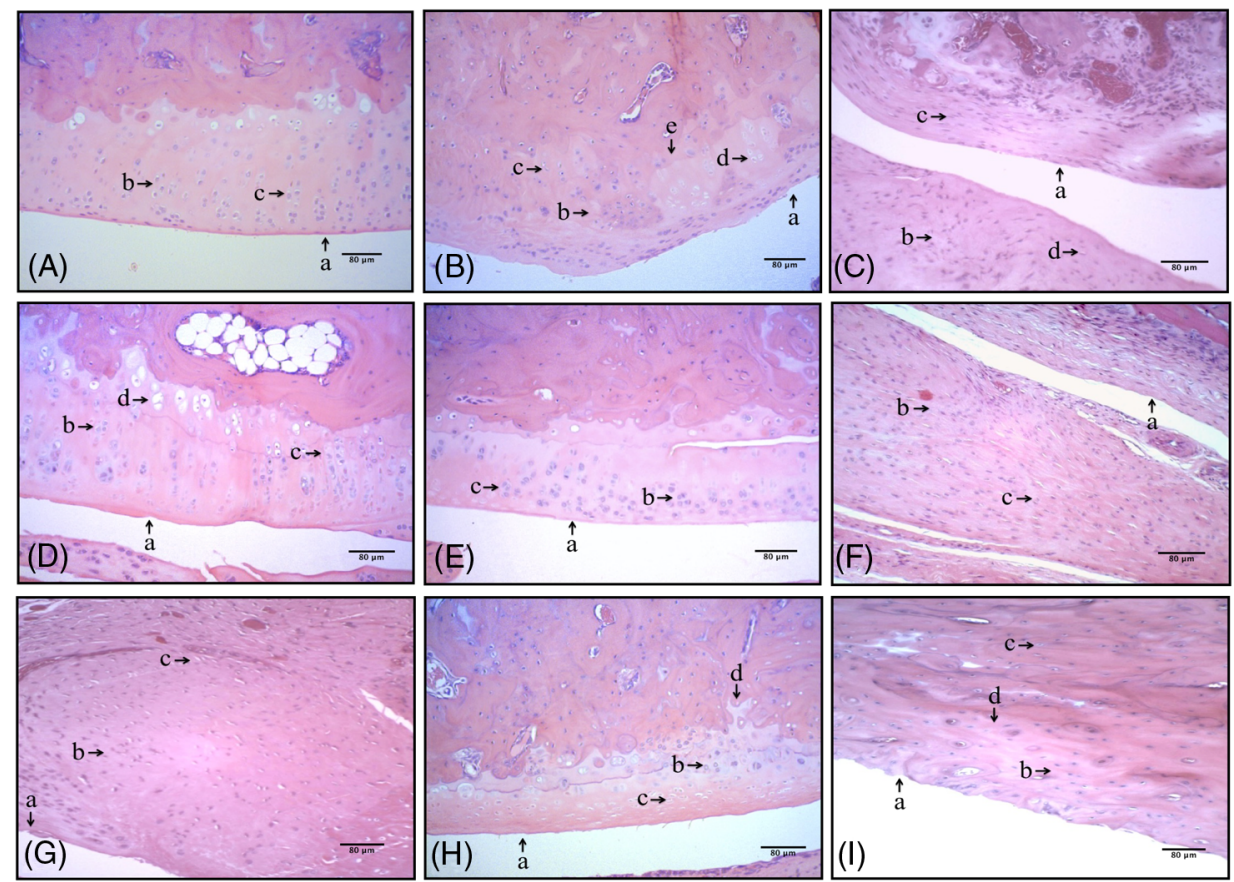

Fig. 1 Histologic characterization of the knee joint. (A) Control group: (a) regular articular surface, (b) chondrocytes distributed homogeneously, and (c) zone of resting cartilage; (B) OA group: (a) irregular articular surface, (b) chondrocytes distributed heterogeneously, (c) zone of resting cartilage, (d) hypertrophic cartilage, and (e) deep invagination of bone tissue; (C) Exerc group: (a) regular articular surface, (b) chondrocytes distributed heterogeneously, (c) homogeneously, (d) zone of resting cartilage; (D) Diclo group: (a) irregular articular surface, (b) chondrocytes distributed heterogeneously, (c) serial cartilage zone, (d) hypertrophic cartilage; (E) PBMT group: (a) regular articular surface, (b) chondrocytes distributed homogeneously, (c) zone of resting cartilage; (F) Exerc + Diclo group: (a) irregular articular surface, (b) chondrocytes distributed heterogeneously, (c) zone of resting cartilage; (G) Exerc + PBMT group: (a) irregular articular surface, (b) chondrocytes distributed heterogeneously, (c) zone of resting cartilage; (H) Diclo + PBMT: (a) irregular articular surface, (b) chondrocytes distributed heterogeneously, (c) zone of resting cartilage, (d) invagination of bone tissue; (I) Exerc + Diclo + PBMT group: (a) irregular articular surface, (b) chondrocytes distributed heterogeneously, (c) zone of resting cartilage, (d) invagination of bone tissue. Magnification 100x.

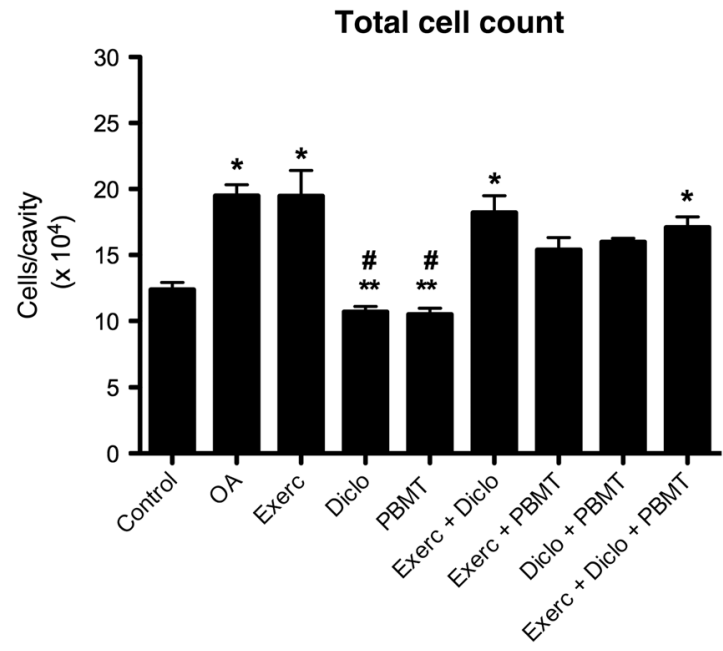

Fig. 2 Total number of cells in the experimental groups. ${ }^{*} p<0.05$ indicates significant difference compared to the control group. $\# p<0.05$ indicates significant difference compared to the OA group. ${ }^{* *} p<0.05$ indicates significant difference compared to the Exerc, Exerc + Diclo, Exerc + PBMT, Diclo + PBMT, and Exerc + Diclo + PBMT groups. Error bars indicate SEM.

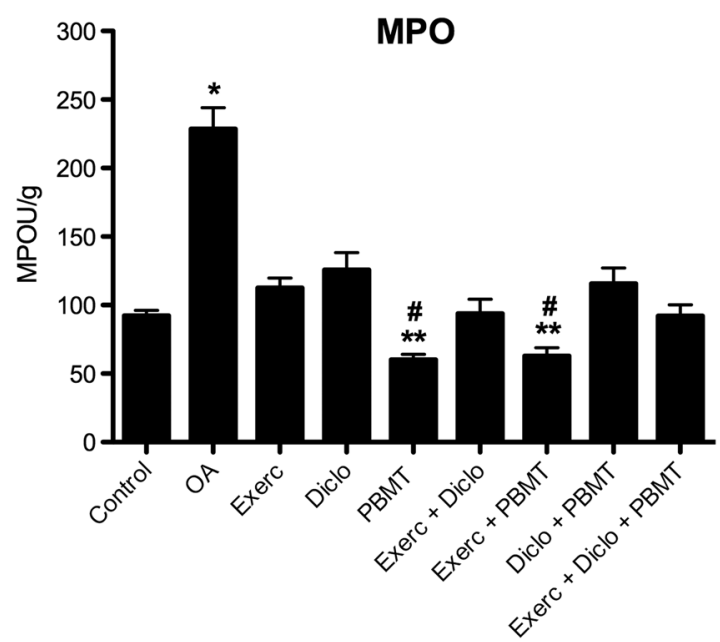

Fig. 3 MPO activity in the experimental groups. ${ }^{*} p<0.05$ indicates significant difference compared to the all experimental groups. $\# p<0.05$ indicates significant difference compared to the control group. ${ }^{* *} p<0.05$ indicates significant difference compared to the Exerc, Diclo, and Diclo + PBMT groups. Error bars indicate SEM. 
(a)

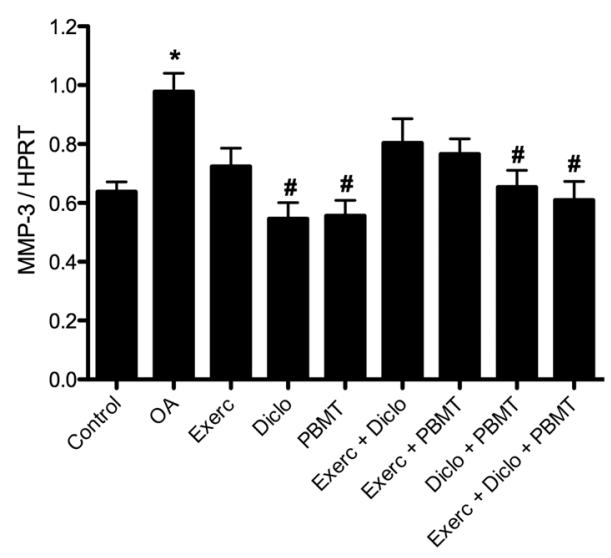

(b)

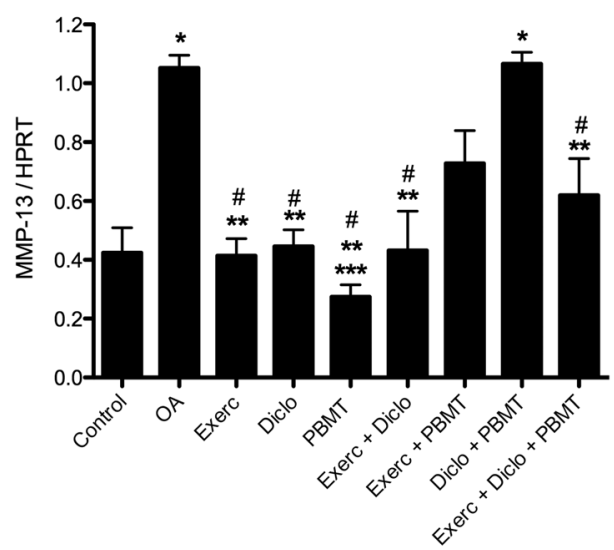

Fig. 4 MMP-3 and MMP-13 gene expression in the experimental groups. (a) ${ }^{*} p<0.05$ indicates significant difference compared to the control group. $\# p<0.05$ indicates significant difference compared to the OA group. (b) ${ }^{\star} p<0.05$ indicates significant difference compared to the control group. $\# p<0.05$ indicates significant difference compared to the OA group. ${ }^{\star *} p<0.05$ indicates significant difference compared to the Diclo + PBMT group. ${ }^{\star \star *} p<0.05$ indicates significant difference compared to the Exerc + PBMT group. Error bars indicate SEM.

In contrast with our results, Assis et al. ${ }^{22}$ observed that physical exercise using treadmills decreased MMP-13 levels. Similarly, Milares et al. ${ }^{23}$ demonstrated that aquatic activities decreased MMP-13 levels and the intensity of the signs of tissue degradation. Although physical exercises are widely prescribed for $\mathrm{OA}$, considerable controversy still exists regarding the best type, intensity, and number of sessions to be performed. ${ }^{24,25}$ Therefore, we believe that the physical exercise parameters used in this study might contribute to the unfavorable results, considering that Assis et al. ${ }^{22}$ and Milares et al. ${ }^{23}$ observed significant alterations with the use of this therapy.

We observed that topical NSAID use was neither effective in modulating the inflammatory process nor in decreasing joint tissue degradation. NSAID therapy has been known to be a treatment option for controlling pain in patients with OA. ${ }^{26}$ However, studies demonstrated that the effectiveness of these drugs for the treatment of this condition is questionable because the results were only slightly better than those obtained with the use of a placebo. ${ }^{8,9,27}$ We observed that the topical use of diclofenac sodium did not improve morphological alterations triggered by our experimental OA model, demonstrating that drug-alternative therapies are promising, which confirmed the findings of the above-mentioned studies.

Furthermore, our results confirmed those reported by Milares et al. ${ }^{23}$ and Assis et al. ${ }^{22}$ who demonstrated that the association of therapies did not produce additive effects. Favorable results from the association of therapies in this study are probably caused by the effects of PBMT because their isolated use has been demonstrated to modulate inflammation and decrease tissue degradation, which were not observed with the isolated use of other therapies.

Our results suggest that, among the therapies tested, the isolated use of PBMT was the most effective for improving the outcomes, indicating the important role of this therapy in $\mathrm{OA}$ treatment. To date, the use of PBMT has not been related to negative side effects or restrictions in musculoskeletal disorders. Therefore, this therapy is considered effective and safe.

We believe that the practice of physical exercises for OA should be further investigated, and effective exercise protocols for modulating the inflammatory process and reducing signs of degradation should be established. Furthermore, our results do not support the association between PBMT, physical exercises, and topical NSAID use because no signs of potential beneficial effects are observed in isolated therapies, and its use would increase the duration and cost of treatment.

\section{Conclusion}

Our results indicate that PBMT used alone is the best alternative among the therapies tested in this study, because it reduces the morphological changes and the enzymes that are involved in joint deterioration. However, further studies are required to examine more specifically the role of physical exercise in OA, and to evaluate the association of therapies on pain and loss of functionality.

\section{Acknowledgments}

Shaiane Silva Tomazoni would like to thank Sao Paulo Research Foundation-FAPESP for PhD scholarship (Grant No. 2011/ 18785-9). The funders had no role in study design, data collection and analysis, decision to publish, or preparation of the manuscript.

\section{References}

1. A. D. Woolf and B. Pfleger, "Burden of major musculoskeletal conditions," Bull. World Health Org. 81(9), 646-656 (2003).

2. Y. Escalante et al., "Physical exercise and reduction of pain in adults with lower limb osteoarthritis: a systematic review," J. Back Musculoskelet. Rehabil. 23(4), 175-186 (2010).

3. K. Kuettner and V. M. Goldberg, "Introduction," in Osteoarthritic Disorders, K. Kuettner and V. M. Goldberg (Eds.), American Academy of Orthopaedic Surgeons, Rosemont, Illinois (1995).

4. S. Krasnokutsky, J. Samuels, and S. B. Abramson, "Osteoarthritis in 2007," Bull. NYU. Hosp. Jt. Dis. 65(3), 222-228 (2007).

5. K. D. Brandt, H. J. Mankin, and L. E. Shulman, "Workshop on etiopathogenesis of osteoarthritis," J. Rheumatol. 13, 1126-1160 (1986).

6. S. M. Seed, K. C. Dunican, and A. M. Lynch, "Treatment options for osteoarthritis: considerations for older adults," Hosp. Pract. 39(1), 62-73 (2011). 
7. W. Zhang, A. Jones, and M. Doherty, "Does paracetamol (acetaminophen) reduce the pain of osteoarthritis? a meta-analysis of randomised controlled trials," Ann. Rheum. Dis. 63(8), 901-907 (2004).

8. J. M. Bjordal et al., "Non-steroidal anti-inflammatory drugs, including cyclo-oxygenase-2 inhibitors, in osteoarthritic knee pain: meta-analysis of randomised placebo controlled trials," BMJ 329(7478), 1317 (2004).

9. H. Tannenbaum et al., "Third Canadian Consensus Conference Group. An evidence-based approach to prescribing nonsteroidal antiinflammatory drugs. Third Canadian Consensus Conference," J. Rheumatol. 33(1), 140-157 (2006).

10. J. M. Hootman et al., "Physical activity levels among the general US adult population and in adults with and without arthritis," Arthritis Rheum. 49(1), 129-135 (2003).

11. R. C. Pallotta et al., "Infrared (810-nm) low-level laser therapy on rat experimental knee inflammation," Lasers Med. Sci. 27(1), 71-78 (2012).

12. E. S. Pessoa et al., "A histologic assessment of the influence of lowintensity laser therapy on wound healing in steroid-treated animals," Photomed. Laser Surg. 22(3), 199-204 (2004).

13. A. C. Alves et al., "Effect of low-level laser therapy on metalloproteinase MMP-2 and MMP-9 production and percentage of collagen types I and III in a papain cartilage injury model," Lasers Med. Sci. 29(3), 911-919 (2013).

14. A. C. Alves et al., "Effect of low-level laser therapy on the expression of inflammatory mediators and on neutrophils and macrophages in acute joint inflammation," Arthritis Res. Ther. 15(5), R116 (2013).

15. A. S. Da Rosa et al., "Effects of low-level laser therapy at wavelengths of 660 and $808 \mathrm{~nm}$ in experimental model of osteoarthritis," Photochem. Photobiol. 88(1), 161-166 (2012).

16. S. A. Dos Santos et al., "Comparative analysis of two low-level laser doses on the expression of inflammatory mediators and neutrophils and macrophages in acute joint inflammation," Lasers Med. Sci. 29(3), 1051-1058 (2014).

17. N. Murat et al., "Quantification of papain-induced rat osteoarthritis in relation to time with the Mankin score," Acta Orthop. Traumatol. Turc. 41(3), 233-237 (2007).

18. W. P. Lima et al., "Lipid metabolism in trained rats: effect of guaraná (Paullinia cupana Mart.) supplementation," Clin. Nutr. 24(6), 10191028 (2005)

19. X. Wang et al., "Overexpression of human matrix metalloproteinase-12 enchances the development of inflammatory arthritis in transgenic rabbits," Am. J. Pathol. 165(4), 1375-1383 (2004).
20. M. J. Steinbeck et al., "Myeloperoxidase and chlorinated peptides in osteoarthritis: potential biomarkers of the disease," J. Orthop. Res. 25(9), 1128-1135 (2007).

21. Y. Oshima et al., "Effect of light-emitting diode (LED) therapy on the development of osteoarthritis (OA) in a rabbit model," Biomed. Pharmacother. 65, 224-229 (2011).

22. L. Assis et al., "Aerobic exercise training and low-level laser therapy modulate inflammatory response and degenerative process in an experimental model of knee osteoarthritis in rats," Osteoarthritis Cartilage 24, 169-177 (2016).

23. L. P. Milares et al., "Effectiveness of an aquatic exercise program and low-level laser therapy on articular cartilage in an experimental model of osteoarthritis in rats," Connect Tissue Res. 57(5), 398-407 (2016).

24. B. A. Egan and J. C. Mentes, "Benefits of physical activity for knee osteoarthritis: a brief review," J. Gerontol. Nurs. 36(9), 9-14 (2010).

25. C. Juhl et al., "Impact of exercise type and dose on pain and disability in knee osteoarthritis: a systematic review and meta-regression analysis of randomized controlled trials," Arthritis. Rheumatol. 66(3), 622-636 (2014).

26. P. Fuller and S. Roth, "Diclofenac sodium topical solution with dimethyl sulfoxide, a viable alternative to oral nonsteroidal anti-inflammatories in osteoarthritis: review of current evidence," J. Multidiscip. Healthcare 4, 223-231 (2011).

27. J. M. Bjordal, "Evidence-based medicine turned upside down," Photomed. Laser Surg. 33(8), 391-392 (2015).

Rodrigo Álvaro Brandão Lopes-Martins is a director of the Center for Technological Research and of the postgraduate program in biomedical engineering at the University of Mogi das Cruzes-UMC. He is a biologist, with a Master in Pharmacology from UNICAMP, and a $\mathrm{PhD}$ in cellular and molecular biology from FIOCRUZ. He is a postdoctoral fellow at the USP. He is a visiting associate professor at the University of Bergen, Norway. He was a professor at the University of Sao Paulo from 2005 to 2015 and an associate professor at the Faculty of Medicine at the Universidade Camilo Castelo BrancoUNICASTELO.

Biographies for the other authors are not available. 